IJMMS 28:11 (2001) 685-688

PII. S016117120100638X

http://ijmms.hindawi.com

(c) Hindawi Publishing Corp.

\title{
ON THE FIXED POINTS OF AFFINE NONEXPANSIVE MAPPINGS
}

\author{
HÜLYA DURU
}

(Received 9 December 2000)

\begin{abstract}
Let $K$ be a closed convex bounded subset of a Banach space $X$ and let $T: K \rightarrow K$ be a continuous affine mapping. In this note, we show that (a) if $T$ is nonexpansive then it has a fixed point, (b) if $T$ has only one fixed point then the mapping $A=(I+T) / 2$ is a focusing mapping; and (c) a continuous mapping $S: K \rightarrow K$ has a fixed point if and only if, for each $x \in K,\left\|\left(A^{n} \circ S\right)(x)-\left(S \circ A^{n}\right)(x)\right\| \rightarrow 0$ for some strictly nonexpansive affine mapping $T$.
\end{abstract}

2000 Mathematics Subject Classification. 47H09, 47H10.

1. Introduction. Let $(X, d)$ be a complete metric space. A continuous mapping $\varphi: X \rightarrow X$ is said to focus at a point $x_{0} \in X$ if, for each $x \in X, d\left(\varphi^{n}(x), x_{0}\right) \rightarrow 0$. Here $\varphi^{n}=\varphi \circ \cdots \circ \varphi$ (n-times). If $\varphi$ focuses at some point $x_{0}$ then $\varphi$ is said to be a focusing mapping. As is well known, every contraction mapping $\varphi: X \rightarrow X$ focuses at its fixed point. However not every focusing mapping is contraction. For example, if $(X, d)$ is compact and $\varphi$ is strictly nonexpansive (i.e., $d(\varphi(x), \varphi(y))<d(x, y)$ for each $x \neq$ $y$ ), then $\varphi$ is not necessarily a contraction but it focuses at its unique fixed point [2]. The importance and relevance of the focusing mappings are due to the fact that a continuous mapping $T$ from a closed convex subset $K$ of a Banach space $(X,\|\cdot\|)$ into itself has a fixed point if and only if there exists a focusing mapping $\varphi: K \rightarrow K$ such that $\lim _{n \rightarrow \infty}\left\|\left(\varphi^{n} \circ T\right)(x)-\left(T \circ \varphi^{n}\right)(x)\right\|=0$ for each $x \in K$ [2]. However a continuous mapping $\varphi: K \rightarrow K$ having a unique fixed point $x_{0} \in K$ need not to focus at this point. Indeed, if we take $X=\ell^{2}$ and $T: X \rightarrow X$ is given, for $x=\left(x_{1}, x_{2}, \ldots, x_{n}, \ldots\right)$, by $T(x)=\left(0, x_{1}, x_{2}, \ldots, x_{n}, \ldots\right)$, then $\varphi=T_{\mid K}$, the restriction of $T$ to the closed unit ball $K$ of $\ell^{2}$, applies $K$ into itself, has only one fixed point, namely $x_{0}=(0,0,0, \ldots)$, but it is not a focusing mapping since, for every $x \in K$ and $n \in \mathbb{N},\left\|\varphi^{n}(x)-x_{0}\right\|_{2}=\|x\|_{2}$. Observe that this mapping $\varphi$ is affine and nonexpansive (i.e., for each $x, y \in K$ and $0 \leq \lambda \leq 1, T(\lambda x+(1-\lambda) y)=\lambda T(x)+(1-\lambda) T(y)$ and $\|\varphi(x)-\varphi(y)\| \leq\|x-y\|)$. In the present paper, our aim is to show that (a) every nonexpansive affine mapping $T$ defined on a convex closed bounded subset $K$ of a Banach space $(X ;\|\|)$ has at least one fixed point, (b) the mapping $A=(I+T) / 2$ is a focusing mapping if and only if $T$ has a unique fixed point; and (c) a continuous mapping $S: K \rightarrow K$ has a fixed point if and only if, for each $x \in K,\left\|\left(A^{n} \circ S\right)(x)-\left(S \circ A^{n}\right)(x)\right\| \rightarrow 0$ for some strictly nonexpansive affine mapping $T: K \rightarrow K$.

2. Results. Throughout, we will assume that $(X,\|\cdot\|)$ is a Banach space, $K$ closed convex bounded subset of $X$, and $T: K \rightarrow K$ is a continuous mapping and $A=(I+T) / 2$. 
LEMMA 2.1. If $T$ is a nonexpansive and, for some $x \in K$, the sequence $\left(A^{n}(x)\right)_{n \in \mathbb{N}}$ has a cluster point $x_{0}$ then $x_{0}$ is a fixed point of $T$

Proof. We recall that, $T$ being nonexpansive , for every $y \in K,\left\|A^{n}(y)-A^{n+1}(y)\right\|$ $\rightarrow 0$ [3, page 98]. Observe also that a point $x_{0} \in K$ is a fixed point of $T$ if and only if it is a fixed point of $A$. Now by hypothesis, there exists a subsequence $\left(A^{n_{k}}(x)\right)_{k \in \mathbb{N}}$ of $\left(A^{n}(x)\right)_{n \in \mathbb{N}}$ converging to $x_{0}$. Since $\left\|A^{n}(x)-A^{n+1}(x)\right\| \rightarrow 0$, we have $\| A^{n_{k}}(x)-$ $A^{n_{k}+1}(x) \| \rightarrow 0$. Hence, we have $x_{0}=A\left(x_{0}\right)$, so $x_{0}=T\left(x_{0}\right)$.

If $T$ has a unique fixed point, then it is not necessarily a focusing mapping as mentioned above. Observe that, in the above example, the set $K$ is compact in the weak topology and the mapping $T$, being linear, is continuous in the same topology. The following result gives a necessary and sufficient condition for a mapping defined on a compact convex set to be focusing.

Proposition 2.2. If $K$ is compact then $T$ is focusing if and only if $T$ has a unique fixed point and for each $x \in K,\left\|T^{n}(x)-T^{n+1}(x)\right\| \rightarrow 0$.

Proof. Suppose that $x_{0}$ is the unique fixed point of $T$ and that for each $x \in K$, $\left\|T^{n}(x)-T^{n+1}(x)\right\| \rightarrow 0$. Fix a point $x \in K$. Then, since $K$ is compact, the sequence $\left(T^{n}(x)\right)_{n \in \mathbb{N}}$ has a convergent subsequence $\left(T^{n_{k}}(x)\right)_{k \in \mathbb{N}}$ converging to some point $y_{0}$ in $K$. Hence, $T$ being continuous, $T^{n_{k}+1}(x) \rightarrow T\left(y_{0}\right)$. Since $\left\|T^{n}(x)-T^{n+1}(x)\right\| \rightarrow 0$, $\left\|T^{n_{k}}(x)-T^{n_{k}+1}(x)\right\| \rightarrow 0$ too. So $T\left(y_{0}\right)=y_{0}$. The fixed point of $T$ being unique, we conclude that $y_{0}=x_{0}$. Hence $x_{0}$ is the only cluster point of the sequence $\left(T^{n}(x)\right)_{n \in \mathbb{N}}$. The set $K$ being compact, the whole sequence $\left(T^{n}(x)\right)_{n \in \mathbb{N}}$ converges to $x_{0}$. This shows that $T$ focuses at $x_{0}$. Conversely, if $T$ focuses at $x_{0}$, for each $x \in K$, the sequence $\left(T^{n}(x)\right)_{n \in \mathbb{N}}$ converges to $x_{0}$; so does $\left(T^{n+1}(x)\right)_{n \in \mathbb{N}}$. As $T$ is continuous, $T\left(x_{0}\right)=x_{0}$ so that $x_{0}$ is the unique fixed point of $T$ and $\left\|T^{n}(x)-T^{n+1}(x)\right\| \rightarrow\left\|x_{0}-x_{0}\right\|=0$.

The next corollary is immediate by Lemma 2.1.

COROLLARY 2.3. If $K$ is compact, $T$ is nonexpansive and has a unique fixed point $x_{0}$, then $A$ focuses at $x_{0}$.

The following result is the main result of the paper. The reader will remark that we do not assume that the set $K$ is compact.

THEOREM 2.4. If $T$ is nonexpansive and affine then it has a fixed point.

Proof. Since $T$ is affine, for each $x \in K$,

$$
A^{n}(x)=\frac{1}{2^{n}}\left[x+\left(\begin{array}{c}
n \\
1
\end{array}\right) T(x)+\cdots+T^{n}(x)\right] .
$$

From here we get

$$
\begin{aligned}
\left\|A(x)-A^{2}(x)\right\| & =\frac{1}{2^{2}}\left\|x-T^{2}(x)\right\| \leq \frac{1}{2^{2}} \delta(K), \\
\left\|A^{2}(x)-A^{3}(x)\right\| & =\frac{1}{2^{3}}\left\|x+T(x)-T^{2}(x)-T^{3}(x)\right\| \leq \frac{1}{2^{3}} 2 \delta(K), \ldots,
\end{aligned}
$$


and so on. By induction on $n$, we obtain

$$
\begin{aligned}
& \left\|A^{2 n+1}(x)-A^{2 n+2}(x)\right\| \leq \frac{1}{2^{2 n+2}}\left(\begin{array}{c}
2 n+1 \\
n
\end{array}\right) \delta(K), \\
& \left\|A^{2 n+2}(x)-A^{2 n+3}(x)\right\| \leq \frac{1}{2^{2 n+3}}\left(\begin{array}{c}
2 n+2 \\
n+1
\end{array}\right) \delta(K) .
\end{aligned}
$$

Here $\delta(K)$ denotes the diameter of $K$. Hence, by the inequality

$$
\left\|A^{2 n+1}(x)-A^{2 n+3}(x)\right\| \leq\left\|A^{2 n+1}(x)-A^{2 n+2}(x)\right\|+\left\|A^{2 n+2}(x)-A^{2 n+3}(x)\right\|,
$$

we have

$$
\left\|A^{2 n+1}(x)-A^{2 n+3}(x)\right\| \leq \frac{1}{2^{2 n+3}}\left[2\left(\begin{array}{c}
2 n+1 \\
n
\end{array}\right)+\left(\begin{array}{c}
2 n+2 \\
n+1
\end{array}\right)\right] \delta(K) .
$$

Moreover by induction on $n$, one can easily see that

$$
\frac{1}{2^{2 n+3}}\left[2\left(\begin{array}{c}
2 n+1 \\
n
\end{array}\right)+\left(\begin{array}{c}
2 n+2 \\
n+1
\end{array}\right)\right] \leq\left(\frac{3}{4}\right)^{n+1} .
$$

Thus

$$
\left\|A^{2 n+1}(x)-A^{2 n+3}(x)\right\| \leq\left(\frac{3}{4}\right)^{n+1} \delta(K) .
$$

Also, by a similar argument, we also have

$$
\left\|A^{2 n}(x)-A^{2 n+2}(x)\right\| \leq\left(\frac{3}{4}\right)^{n} \delta(K) .
$$

It follows from these inequalities that the sequences $\left(A^{2 n}(x)\right)_{n \in \mathbb{N}}$ and $\left(A^{2 n+1}(x)\right)_{n \in \mathbb{N}}$ are Cauchy sequences. So they converge. As $\left\|A^{n}(x)-A^{n+1}(x)\right\| \rightarrow 0$, the sequences $\left(A^{2 n}(x)\right)_{n \in \mathbb{N}}$ and $\left(A^{2 n+1}(x)\right)_{n \in \mathbb{N}}$ converge to the same point. Hence the sequence $\left(A^{n}(x)\right)_{n \in \mathbb{N}}$ converges to this point, which is a fixed point of $A$, so of $T$.

At this point we remark that if the set $K$ is not bounded then this theorem fails even if $X$ is finite dimensional. Indeed, let $X=R, K=[0, \infty[$ and $T(x)=x+1$. This mapping is nonexpansive and affine but does not have a fixed point.

From this theorem we conclude that if $T$ is strictly nonexpansive and affine, then $A$ is focusing if and only if it has a unique fixed point. However as mentioned above an affine mapping may have a unique fixed point even if it is not strictly nonexpansive.

Now by the preceding theorem and corollary, the next result is immediate.

COROLlary 2.5. If $T$ is nonexpansive, affine, and has a unique fixed point, say $x_{0}$, then $A$ is a focusing mapping and focuses at its fixed point.

As applications of these results we present the following.

Proposition 2.6. Let $S: K \rightarrow K$ be a continuous mapping. Then $S$ has a fixed point if and only if there exists a strictly nonexpansive affine mapping $T: K \rightarrow K$ such that, for each $x \in K,\left\|A^{n} \circ S(x)-S \circ A^{n}(x)\right\| \rightarrow 0$. 
Proof. Let $x_{0}$ be the fixed point of $S$. Let $T$ be the constant mapping $T(x)=x_{0}$, which is affine and strictly nonexpansive. Then $A(x)=\left(x+x_{0}\right) / 2$, and, as a direct simple calculation shows, $A^{n}(x) \rightarrow x_{0}$ for each $x \in K$, so that $\left\|A^{n} \circ S(x)-S \circ A^{n}(x)\right\| \rightarrow 0$. Conversely, if, for some strictly nonexpansive affine mapping $T: K \rightarrow K$, we have $\left\|A^{n} \circ S(x)-S \circ A^{n}(x)\right\| \rightarrow 0$ (we recall that $A=(I+T) / 2$ ) then, by Theorem 2.4, $T$ has a fixed point $x_{0}$ and since $T$ is strictly nonexpansive, $x_{0}$ is the unique point of $T$. So by Corollary 2.5, $A$ focuses at $x_{0}$. Hence $\left\|A^{n} \circ S(x)-S \circ A^{n}(x)\right\| \rightarrow\left\|x_{0}-S\left(x_{0}\right)\right\|=0$, which completes the proof.

Remark that Proposition 2.6 remains valid if $T$ is not strictly nonexpansive but nonexpansive and has a unique fixed point. This remark also applies to the next result.

The classical Markov-Kakutani theorem [1, page 456] states that a family of continuous affine and pairwise commuting family of mappings from a convex compact subset of a locally convex topological vector space into itself has a common fixed point. In the next result we show that, in the setting of Banach spaces, dropping compactness and assuming that one of the mapping is strictly nonexpansive, we still have the same conclusion.

COROLLARY 2.7. Let $\left(T_{\alpha}\right)_{\alpha \in I}$ be a family of pairwise commuting nonexpansive affine mappings on $K$. If one of them, say $T_{\alpha_{0}}$, is strictly nonexpansive, then the family $\left(T_{\alpha}\right)_{\alpha \in I}$ has a unique common fixed point.

Proof. By Theorem 2.4, $T_{\alpha_{0}}$ has a unique fixed point $x_{0}$. Since the mappings $T_{\alpha}^{\prime} s$ commute pairwise, $x_{0}$ is a common fixed point of all mappings $T_{\alpha}^{\prime} s$. The uniqueness is obvious.

Acknowledgement. This research was supported by the Research Fund of the University of Istanbul, Project number 1499/28082000.

\section{REFERENCES}

[1] N. Dunford and J. T. Schwartz, Linear Operators. I. General Theory, Pure and Applied Mathematics, vol. 7, Interscience Publishers, New York, 1958. MR 22\#8302. Zbl 0084.10402.

[2] H. Duru, A note on the existence of fixed points of nonexpansive mappings, in preparation.

[3] K. Goebel and W. A. Kirk, Topics in Metric Fixed Point Theory, Cambridge Studies in Advanced Mathematics, vol. 28, Cambridge University Press, Cambridge, 1990. MR 92c:47070. Zbl 0708.47031.

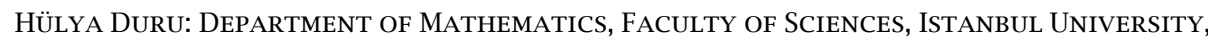
34459 VEZNECILER, ISTANBUL, TURKEY

E-mail address: hduru@istanbu 1.edu.tr 


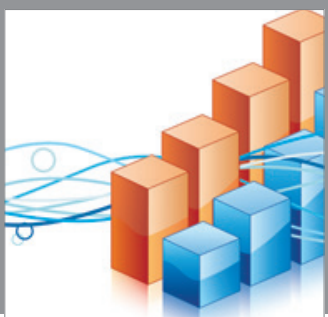

Advances in

Operations Research

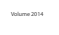

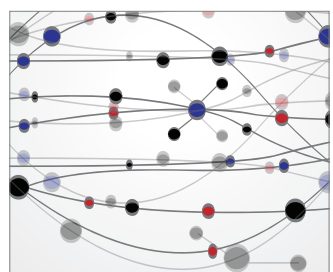

\section{The Scientific} World Journal
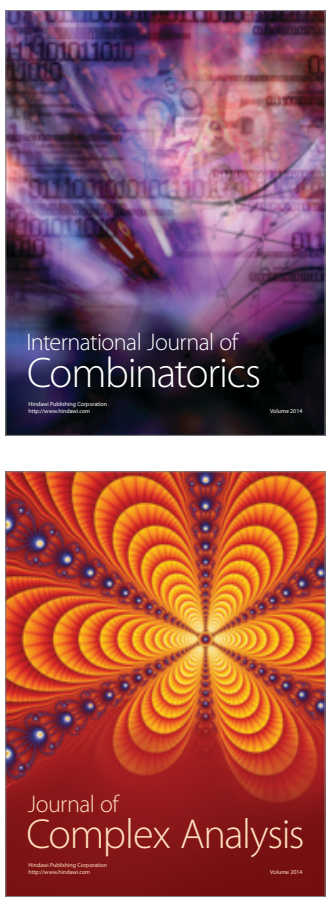

International Journal of

Mathematics and

Mathematical

Sciences
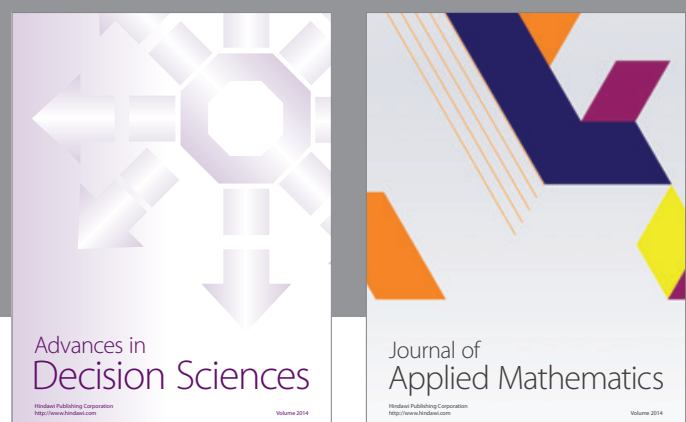

Journal of

Applied Mathematics
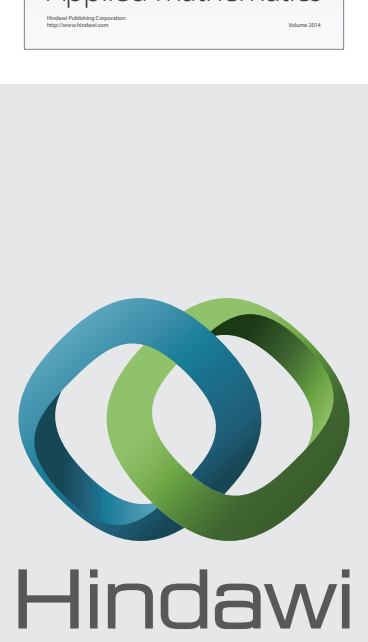

Submit your manuscripts at http://www.hindawi.com
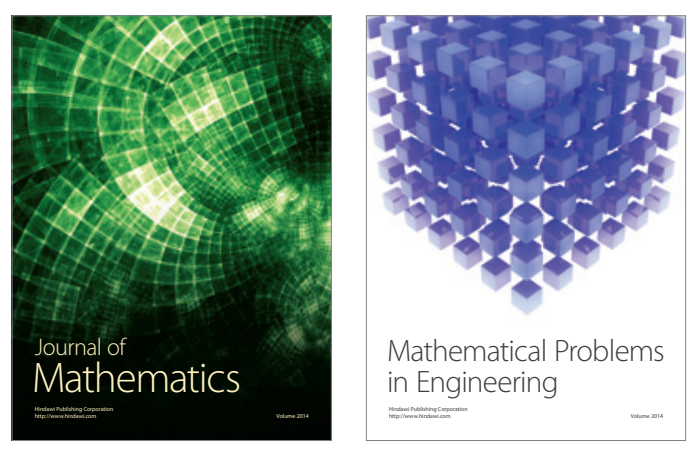

Mathematical Problems in Engineering
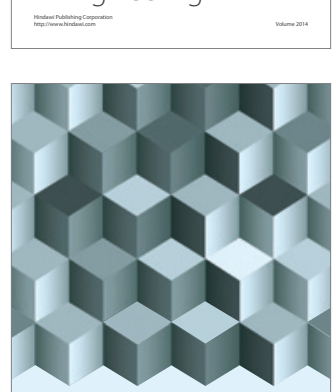

Journal of

Function Spaces
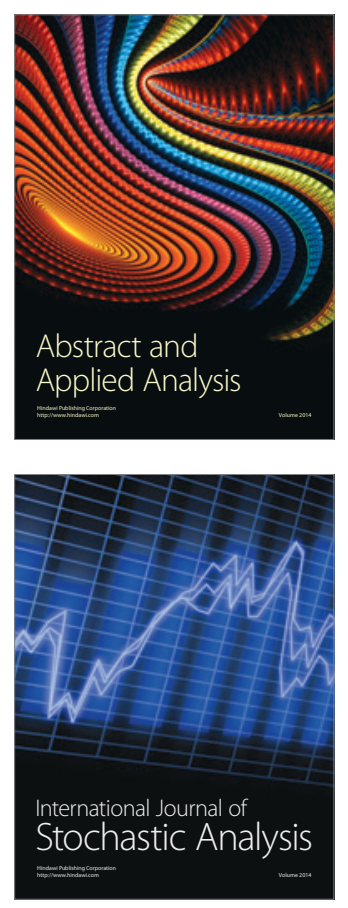

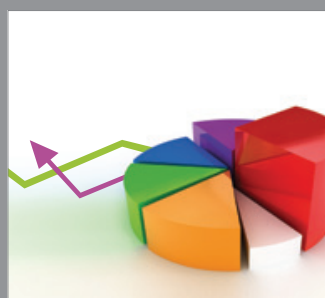

ournal of

Probability and Statistics

Promensencen
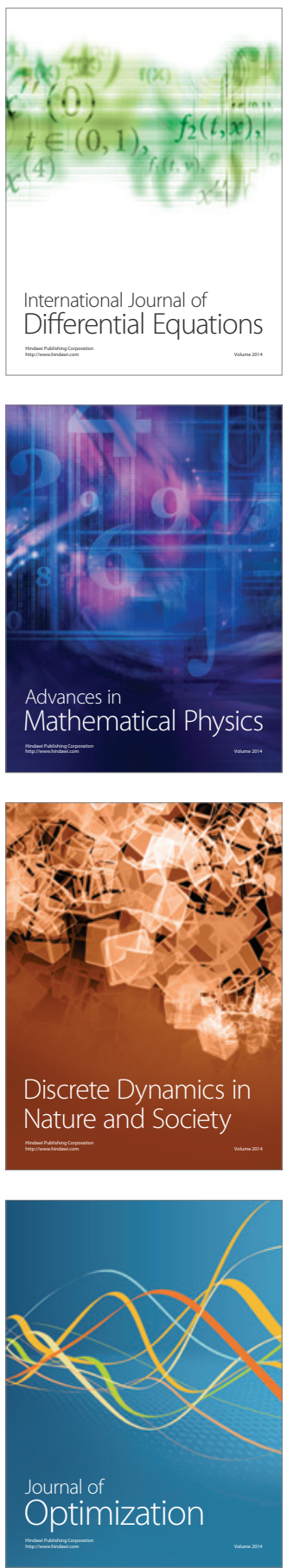